\title{
Phosphorylation of Bcl-2 plays an important role in glycochenodeoxycholate-induced survival and chemoresistance in $\mathrm{HCC}$
}

\author{
MAOJUN ZHOU ${ }^{1}$, QI ZHANG ${ }^{2}$, JINFENG ZHAO ${ }^{3}$, MINGMEI LIAO $^{3}$, SAILAN WEN $^{4}$ and MANYI YANG ${ }^{3}$ \\ ${ }^{1}$ Key Laboratory of Cancer Proteomics of Chinese Ministry of Health, ${ }^{2}$ Department of Hepatobiliary and \\ Pancreatic Surgery, ${ }^{3}$ Key Laboratory of Nanobiological Technology of Chinese Ministry of Health, \\ Xiangya Hospital, Central South University, Changsha, Hunan 410008; ${ }^{4}$ Department of Pathology, \\ The Second Xiangya Hospital, Central South University, Changsha, Hunan 410011, P.R. China
}

Received December 6, 2016; Accepted June 22, 2017

DOI: $10.3892 /$ or.2017.5830

\begin{abstract}
Hepatocellular carcinoma (HCC) is a highly malignant tumor and can evolve rapidly to resistance to chemotherapies. Glycochenodeoxycholate (GCDA), which is toxic and hydrophobic, is the main ingredient in the bile and associated with carcinogenesis of gastrointenstinal tumors. Bcl-2 is the most important anti-apoptotic protein and overexpressed in various human tumors. In the present study, we found that GCDA can induce the chemoresistance of human liver cancer cells and specific depletion of Bcl-2 by RNA interference blocks GCDA-stimulated chemoresistance, which indicate the pivotal role of $\mathrm{Bcl}-2$ in such process. Mechanistically, GCDA simultaneously stimulates phosphorylation of Bcl-2 at Ser70 site and activates extracellular signal-regulated kinase $1 / 2$ (ERK1/2), and inhibition of ERK1/2 by PD98059 (MAPK/ERK1/2 inhibitor) or siRNA (targeting ERK1/2) suppresses GCDA-stimulated phosphorylation of Bcl-2 and significantly attenuates the survival and chemoresistance induced by GCDA in liver cancer cells. Thus, GCDA-induced survival and chemoresistance of liver cancer cells may occur through activation of Bcl-2 by phosphorylation at Ser70 site through MAPK/ERK1/2 pathway, which may contribute to the development of human liver cancer and chemoresistance.
\end{abstract}

\section{Introduction}

The inventory of cancer research of 2015 shows that liver cancer is a global high incidence malignant tumor, which is

Correspondence to: Dr Manyi Yang, Key Laboratory of Nanobiological Technology of Chinese Ministry of Health, Xiangya Hospital, Central South University, 87 Xiangya Road, Changsha, Hunan 410008, P.R. China

E-mail: manyiyang510@163.com

Key words: hepatocellular carcinoma, glycochenodeoxycholate, B-cell leukemia-2, phosphorylation, MAPK/ERK1/2 pathway known as 'the king of cancer' (1). China is the country with high incidence of liver cancer. Chinese population accounts for $1 / 5$ of the world, while the incidence and mortality of liver cancer is more than $1 / 2$ of the world. World's Cancer Report of 2014 shows that hepatocellular carcinoma (HCC) has become the fifth ranked cancer in men ( 554,000 new cases each year) and the ninth cancer in women $(\sim 228,000$ new cases each year) (2). The high prevalence and mortality rate of HCC (the overall mortality rate is $95 \%$ ) make it the major health burden in the current society.

Bile acids are the main component of the bile secreted by liver cells, while conjugated bile acids is the primary form. Due to the $\mathrm{pH}$ environment around the bile, most of which exists as sodium or potassium salt, called bile salts (sodium salt combined with bile acids). Glycochenodeoxycholate (Glycine conjugate of chenodexycholate) is the main ingredient in the bile, which is considered to be toxic and hydrophobic and may be involved in mediating apoptosis of liver cells during cholestasis. Previous studies have shown that toxic bile salt glycochenodeoxycholate induces hepatocyte apoptosis involving ligand-independent oligomerization of Fas (3) and by promoting cytoplasmic transport of Fas to the cell surface (4). PKC-dependent signaling pathways play a critical role in bile salt-induced hepatocyte apoptosis $(5,6)$. Endoplasmic reticulum is involved in glycochenodeoxycholic acid-induced apoptosis in rat hepatocytes, although its role may be smaller than mitochondria-mediated pathway (7). Another study demonstrated that glycochenodeoxycholate induces apoptosis of liver cells by modulating the IGF1 system (8). Although the accumulation of glycochenodeoxycholate induced hepatocyte apoptosis in cholestasis, many hepatocytes still survive. The study by Wang et al (9) found that glycochenodeoxycholateinduced caspase response is reversible, which may activate anti-apoptotic genes to protect hepatocytes from apoptosis. Later, another study by Wang et al (10) demonstrated an interesting result: a low dosage of glycochenodeoxycholate induced hepatocyte apoptosis to exhibit the biphasic response, which was regulated by the expression of survivin through $N F-\kappa B$ signaling pathway. Recent research suggests that PKC- $\delta$ activation by glycochenodeoxycholate stimulates 
a cytoprotective pathway involving JNK inhibition, Akt activation and downregulation of BIM (11). Moreover, research has confirmed that bile salt, especially toxic bile salt, is associated with carcinogenesis of gastrointenstinal tumors. Bile acids can block Mcl-1 protein degradation via activation of an EGFR/Raf-1 cascade resulting in tumorigenesis of cholangiocarcinomas (12). Glycochenodeoxycholic acid activates the PI3 Kinase/Akt signaling pathway, stimulate cell growth in the Barrett's adenocarcinoma cell line SEG-1 (13) and induces proliferation of a non-neoplastic Barrett's cell line by activation of both ERK and p38 MAPK pathways (14), which suggest potential mechanisms whereby bile reflux may facilitate the neoplastic progression of Barrett's esophagus. In addition, long-term effect of oxidative DNA damage caused by glycochenodeoxycholate may promote carcinogenesis in the biliary tract (15). The disturbance of bile circulation metabolism may cause abnormal concentration of bile salts, which will result in necrosis and apoptosis of liver cells and become the potential carcinogenesis of hepatocellular carcinoma (HCC). However, the underlying intracellular signaling mechanism remains to be further studied.

Bcl-2 (B Cell Leukemia-2) family proteins are key players in the control of mitochondria-based apoptosis $(16,17)$. Bcl-2 is the most important anti-apoptotic protein, whose overexpression and phosphorylation may be involved in regulating cell proliferation, cell cycle, DNA repair, tumorigenesis and chemoresistance. There is high level of Bcl-2 protein in many human tumors, such as liver, prostate, colon, lung, stomach, lymphoma, neuroblastoma and breast cancer $(17,18)$. Endogenous Bcl-2 expressed in various cells can be phosphorylated at multiple sites in the flexible loop domain (FLD), including Thr69, Ser70 and Ser87, associated with regulation of apoptosis (19). Studies verified that PKC $\alpha$, p44 MAPK/ERK1, p42 MAPK/ERK2 and JNKs are Bcl-2 upstream kinases $(20,21)$. In recent years, Bcl-2's application in clinic is quite common. Bcl-2 may play an important role in mediating the outcome of antidepressant treatment (22). The expression of Bcl-2 may serve as predictive biomarker of response to induction chemotherapy in HNSCC (head and neck squamous cell carcinomas) patients (23). The genetic variation in Bcl-2 3'-UTR was associated with a decreased lung cancer risk and better survival for non-small cell lung cancer in male Chinese (24). Overexpression of Bcl-2 protein predicts chemoresistance in acute myeloid leukemia (25). Genetic polymorphisms in the Bcl-2 gene may be associated with the risk of endometrial cancer in Chinese women (26). Some studies found that inhibitors and microRNA targeting Bcl-2 had effect on treatment of gliomas (27), myeloma (28), nasopharyngeal carcinoma (29), esophageal squamous cell carcinoma (30), aclcoholic liver disease (31), colorectal (32), gastric cancer (33) and melanoma (34).

However, studies on the role of Bcl-2 in hepatocellular carcinoma (HCC) are scarce. The earliest study demonstrated that normal hepatocytes did not express Bcl-2 and expression of Bcl-2 by hepatocytes during cholestasis suggested an adaptive phenomenon to resist apoptosis by toxic bile salts (35). Hepatocytes in the bile duct ligation (BDL) rats expressed Bcl-2, which can inhibit the bile salt-induced hepatocellular apoptosis (36). Related research found that glycochenodeoxycholate (GCDA) may induce the phosphorylation of Mcl-1 (one of the anti-apoptotic member in Bcl-2 family) at T163 site, eventually leading to survival and chemoresistance in hepatocellular carcinoma cells (37). Since GCDA can activate ERK1/2, which is a physiologic Ser70 site kinase of Bcl-2, in the present study, we have shown that GCDA induces Bcl-2 phosphorylation at Ser70 through activation of ERK1/2, which leads to enhanced anti-apototic function in human liver cancer cells.

\section{Materials and methods}

Materials. Sodium glycochenodeoxycholate (GCDA, G0759) was purchased from Sigma-Aldrich (St. Louis, MO, USA). 5-Fluorouracil was purchased from Shanghai Xudong Haipu Pharmaceutical Co.,Ltd. (Shanghai, China). PD98059 (513001) was obtained from Calbiochem (San Diego, CA, USA). Bcl-2 (50E3) rabbit (2870) and phospho-Bcl-2 (Ser70) (5H2) rabbit (2827) antibodies were from Cell Signaling Technology (Danvers, MA, USA). Bcl-2 (N-19) (sc-492), p-Bcl2 (74.Ser70) (sc-135757), ERK1 (K-23) (sc-94), ERK2 (C-14) (sc-154) and p-ERK (E-4) (sc-7383) antibodies were purchased from Santa Cruz Biotechnology (Santa Cruz, CA, USA). Alexa Fluor ${ }^{\circledR}$ 488 goat anti-rabbit $\operatorname{IgG}(\mathrm{H}+\mathrm{L})$ antibody (A-11008), Alexa Fluor ${ }^{\circledR} 594$ goat anti-rabbit $\operatorname{IgG}(\mathrm{H}+\mathrm{L})$ antibody (A-11012) and Alexa Fluor ${ }^{\circledR} 594$ donkey anti-mouse $\operatorname{IgG}(\mathrm{H}+\mathrm{L})$ antibody (A-21203) were obtained from Molecular Probes (Eugene, OR, USA). Monoclonal anti-Flag antibody (F1804) was from Sigma-Aldrich. The mitochondrial marker (Mito-GFP) and endoplasmic reticulum marker (ERM-RFP) plasmids were kindly provided by Dr Yuan (Sun Yat-sen University, Guangzhou, China). All reagents used were obtained from commercial sources.

Cell lines and culture conditions. Five HCC cell lines HepG2, Bel7402, QGY7703, SMMC7721 and Huh7 were purchased from the Institute of Biochemistry and Cell Biology (Shanghai Institutes for Biological Sciences, CAS, Shanghai, China). HepG2, Bel7402, QGY7703 and SMMC7721 were maintained in RPMI-1640 supplemented with $10 \%$ fetal bovine serum (FBS). Huh7 was maintained in Dulbecco's modified Eagle's medium (DMEM) supplemented with 10\% FBS. All of the cell lines were cultured at $37^{\circ} \mathrm{C}$ in a humid incubator with $5 \% \mathrm{CO}_{2}$.

siRNA and transfections. For RNA interference, siRNA specific to Bcl-2 (5'-AAC AUC GCC CUG UGG AUG ACU-3'), ERK1 (5'-GAC CGG AUG UUA ACC UUU ATT-3'), ERK2 (5'-CAC CAA CCA UCG AGC AAA UTT-3') and non-specific control siRNA (5'-UUC UCC GAA CGU GUC ACG UTT-3') were purchased from Shanghai GenePharma, Co., Ltd., (Shanghai, China) siRNAs were transfected into the cells using Lipofectamine RNAi max (Invitrogen) according to the manufacturer's instruction.

Cell viability assay. Cells treated with GCDA, drugs and inhibitors were harvested and washed with phosphate-buffered saline (PBS). Cells were resuspended in binding buffer and stained with Annexin $\mathrm{V}$ and propidium iodide (PI) according to manufacturer's instructions (BD Biosciences Annexin V kit; BD Biosciences, San Diego, CA, USA). 

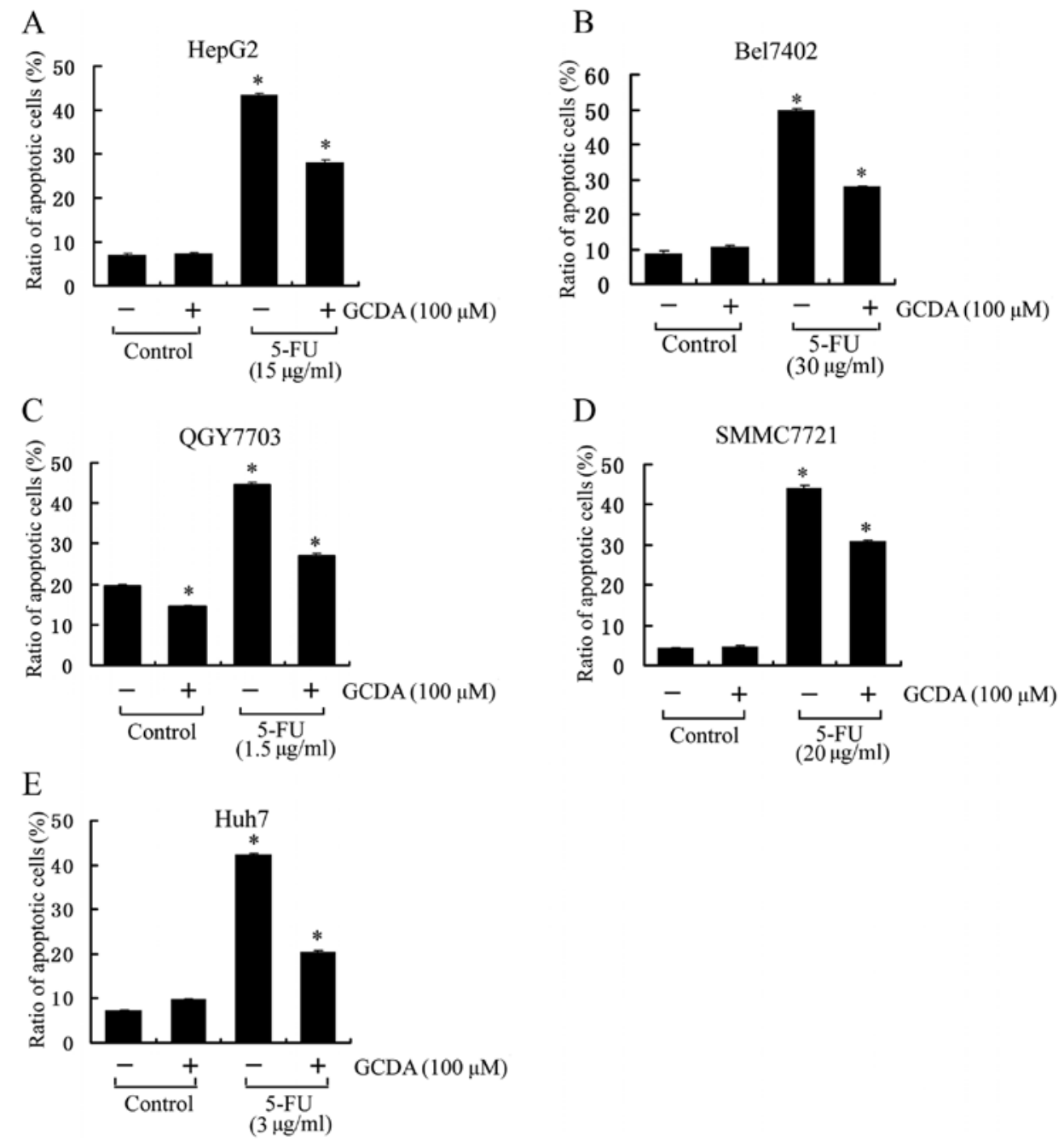

Figure 1. GCDA induces chemoresistance of liver cancer cells. (A) HepG2, (B) Bel7402, (C) QGY7703, (D) SMMC7721 and (E) Huh7 cells were treated with 5-FU with respective $\mathrm{IC}_{50}$ concentration $(15 \mu \mathrm{g} / \mathrm{ml}$ for $\mathrm{HepG} 2,30 \mu \mathrm{g} / \mathrm{ml}$ for Bel7402, $1.5 \mu \mathrm{g} / \mathrm{ml}$ for QGY7703, $20 \mu \mathrm{g} / \mathrm{ml}$ for SMMC7721 and $3 \mu \mathrm{g} / \mathrm{ml}$ for Huh7) in the presence or absence of GCDA $(100 \mu \mathrm{M})$ for $72 \mathrm{~h}$. Cell viability and apoptosis were determined by analyzing Annexin V binding on fluorescenceactivated cell sorting (FACS). Columns, mean of three separate determinations; bars, SD; ${ }^{*} \mathrm{P}<0.05$ (Student's t-test).

Cell extraction and western blotting. After the treatment, the cells were washed with cold PBS and then were removed to $1.5 \mathrm{ml}$ Eppendorf tubes using a cell scraper. The whole-cell extracts were prepared in lysis buffer [50 mM Tris, pH 7.4, $150 \mathrm{mM} \mathrm{NaCl}, 1 \%$ Nonidet P-40, $0.5 \%$ deoxycholic acid sodium salt and cocktail protease inhibitor (Roche)], which were put on ice for $30 \mathrm{~min}$ and then centrifuged at $14,000 \mathrm{rpm}$ for $2 \mathrm{~min}$. The resulting supernatant was collected as the total cell lysate. Aliquots containing $30 \mu \mathrm{g}$ of total protein from each sample were subjected to $10 \%$ SDS-PAGE and electrotransferred to nitrocellulose membranes. The membranes were blocked with 5\% skimmed milk in PBST for $2 \mathrm{~h}$, and then incubated with primary antibodies (diluted in PBST containing 2.5\% skimmed milk) at $4^{\circ} \mathrm{C}$ overnight. After the membranes were washed with PBST three times, they were incubated with horseradish peroxidase-conjugated anti-rabbit or anti-mouse secondary antibody for $2 \mathrm{~h}$. Chemiluminescent detection was performed with SuperSignal West Dura Extended Duration Substrate kit (Thermo Fisher Scientific) and the membranes were exposed to X-ray film.
Immunofluorescence. QGY7703 cells were seeded on coverslips $(10 \mathrm{~mm} \times 10 \mathrm{~mm})$ in a 24 -well plate. After $20-24 \mathrm{~h}$, cells were fixed for $15 \mathrm{~min}$ in a $4 \%$ formaldehyde solution, washed three times in PBST (0.05\% Tween-20 in PBS) and blocked with $5 \% \mathrm{BSA}$ in PBST at $4^{\circ} \mathrm{C}$ overnight. They were then incubated with Bcl-2, p-Bcl-2-Ser70 or p-ERK antibodies for $1 \mathrm{~h}$. The cells were washed three times in PBST, and incubated with the Alexa Fluor ${ }^{\circledR} 488$ (green) goat anti-rabbit $\operatorname{IgG}(\mathrm{H}+\mathrm{L})$ antibody and/or Alexa Fluor ${ }^{\circledR}$ 594 (red) goat anti-rabbit IgG (H+L) antibody and/or Alexa Fluor $^{\circledR} 594$ (red) donkey anti-mouse $\operatorname{IgG}(\mathrm{H}+\mathrm{L})$ antibody for $1 \mathrm{~h}$. Following triple washing in PBS, cells were labeled with $0.2 \mu \mathrm{g} / \mathrm{ml}$ DAPIs in PBS for $5 \mathrm{~min}$, and then washed three times in PBS. Cells were mounted in MOWIOL R4-88 reagent (Calbiochem) and photographed with a Carl Zeiss AxioVision 4 microscope.

Statistical analysis. The data were subject to statistical analysis using the SPSS software package (version 17.0). All data were expressed as the means \pm SD of three or more independent experiments. The statistical differences were analyzed by the 

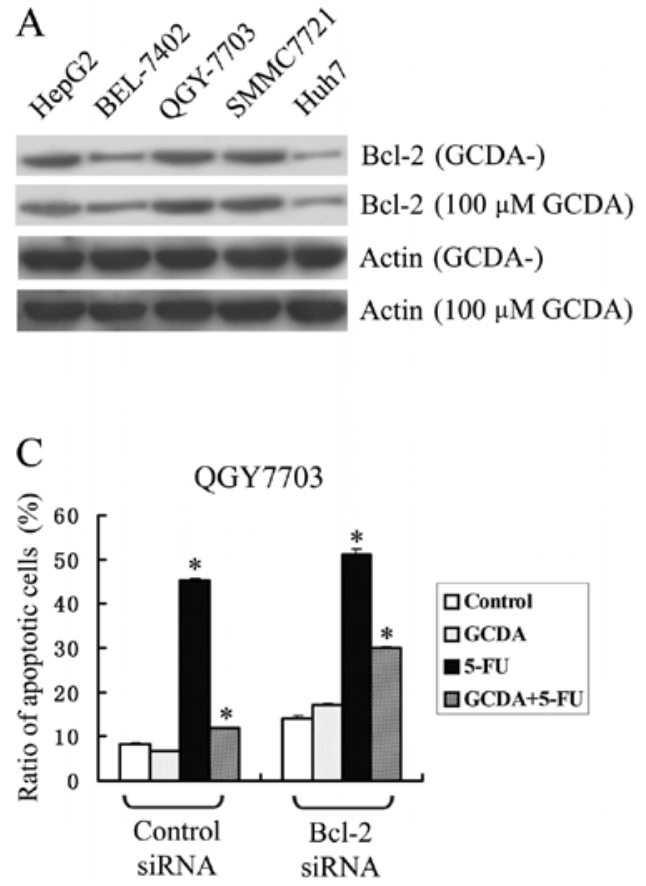
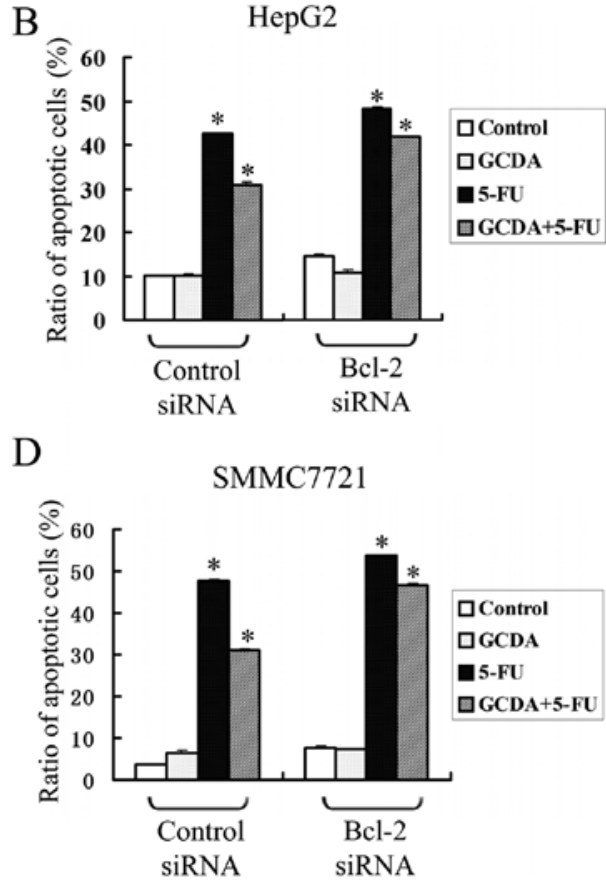

Figure 2. Bcl-2 plays a role in GCDA-induced chemoresistance of liver cancer cells. (A) Expression of Bcl-2 in five liver cancer cell lines (HepG2, Bel7402, QGY7703, SMMC7721 and Huh7) treated with and without $100 \mu \mathrm{M}$ GCDA was analyzed by western blot analysis. (B) HepG2, (C) QGY7703 and (D) SMMC7721 were transfected with siRNA targeting Bcl-2 in the presence of GCDA (100 $\mu \mathrm{M}), 5-\mathrm{FU}$ (15 $\mu \mathrm{g} / \mathrm{ml}$ for HepG2, $1.5 \mu \mathrm{g} / \mathrm{ml}$ for QGY7703 and $20 \mu \mathrm{g} / \mathrm{ml}$ for SMMC7721) or both GCDA $(100 \mu \mathrm{M})$ and 5-FU (15 $\mu \mathrm{g} / \mathrm{ml}$ for HepG2, $1.5 \mu \mathrm{g} / \mathrm{ml}$ for QGY7703 and $20 \mu \mathrm{g} / \mathrm{ml}$ for SMMC7721) for $72 \mathrm{~h}$. Cell viability and apoptosis were determined by analyzing Annexin V binding on fluorescence-activated cell sorting (FACS). Columns, mean of three separate determinations; bars, SD; ${ }^{*} \mathrm{P}<0.05$ (Student's t-test).

Student's t-test. The differences were considered to be statistically significant at $\mathrm{P}<0.05$.

\section{Results}

GCDA induces chemoresistance of human liver cancer cells. 5-Fluorouracil (5-FU) is one of the most important chemotherapy drugs for the advanced liver cancer. However, its clinical efficacy is not satisfactory due to the chemoresistance. The underlying mechanism is not clear yet. In order to test whether bile salt GCDA can induce chemoresistance, five HCC cell lines, HepG2, Bel7402, QGY7703, SMMC7721 and Huh7 were treated with 5 -FU with respective $\mathrm{IC}_{50}$ concentration $(15 \mu \mathrm{g} / \mathrm{ml}$ for HepG2, $30 \mu \mathrm{g} / \mathrm{ml}$ for Bel7402, $1.5 \mu \mathrm{g} / \mathrm{ml}$ for QGY7703, $20 \mu \mathrm{g} / \mathrm{ml}$ for SMMC7721 and $3 \mu \mathrm{g} / \mathrm{ml}$ for Huh7) in the absence or presence of GCDA for $72 \mathrm{~h}$. Cell viability and apoptosis were determined by analyzing Annexin $\mathrm{V}$ binding on fluorescence-activated cell sorting (FACS). Results indicate that in all the five cell lines, 5-FU can induce $~ 50 \%$ of cells to undergo apoptosis and GCDA can significantly attenuate the apoptotic effect of 5-FU (Fig. 1). These findings reveal that GCDA may be one reason for chemoresistance in liver cancer cells. Data represent the mean \pm SD of three determinations. $\mathrm{P}<0.05$ is based on the Student's t-test.

Bcl-2 plays a role in GCDA-induced chemoresistance of human liver cancer cells. Bcl-2 is a major anti-apoptotic member of $\mathrm{Bcl} 2$ family. Consistently, $\mathrm{Bcl}-2$ is widely expressed in human liver cancer cells. Among them, HepG2, QGY7703 and SMMC7721 expressed relatively high levels of endogenous Bcl-2, while Bel7402 and Huh7 expressed relatively low levels of endogenous Bcl-2 (Fig. 2A). After treating the five types of cell-line with $100 \mu \mathrm{M}$ GCDA, the expression levels of endogenous Bcl-2 changed little (Fig. 2A).

To test whether Bcl-2 plays a role in GCDA-induced chemoresistance, three HCC cell-lines with high level of endogenous Bcl-2, HepG2, QGY7703 and SMMC7721 were transfected with siRNA targeting Bcl-2 in the presence of GCDA $(100 \mu \mathrm{M}), 5-\mathrm{FU}$ $(15 \mu \mathrm{g} / \mathrm{ml}$ for HepG2, $1.5 \mu \mathrm{g} / \mathrm{ml}$ for QGY7703 and $20 \mu \mathrm{g} / \mathrm{ml}$ for SMMC7721) or both GCDA $(100 \mu \mathrm{M})$ and 5-FU $(15 \mu \mathrm{g} / \mathrm{ml}$ for HepG2, $1.5 \mu \mathrm{g} / \mathrm{ml}$ for QGY7703 and $20 \mu \mathrm{g} / \mathrm{ml}$ for SMMC7721) for $72 \mathrm{~h}$. Cell viability and apoptosis were determined by analyzing Annexin V binding FACS. Results indicate that in all the three cell-lines, GCDA can attenuate the apoptotic effect of 5-FU. However, after Bcl-2 was silenced by siRNA, the ratio of apoptotic cells was upregulated slightly, and the chemoresistance effect of GCDA was downregulated from 27.23 to $13.41 \%$ in HepG2 cells (Fig. 2B), from 73.86 to $40.93 \%$ in QGY7703 cells (Fig. 2C) and 35.20 to $13.13 \%$ in SMMC7721 cells (Fig. 2D). Data represent the mean $\pm \mathrm{SD}$ of three determinations. $\mathrm{P}<0.05$ is based on the Student's t-test. These findings suggest that $\mathrm{Bcl}-2$ may play a role in GCDA-induced chemoresistance.

GCDA stimulates Bcl-2 phosphorylation at Ser70. According to the results above, GCDA did not affect much the expression of endogenous Bcl-2 (Fig. 2A), but Bcl-2 indeed plays a role in GCDA-induced chemoresistance (Fig. 2B-D). Consistent with previous studies, in QGY7703 cells Bcl-2 localized at mitochondria and endoplasmic reticulum (Fig. 3A and B), which indicates that $\mathrm{Bcl}-2$ may function both at mitochondria and endoplasmic reticulum (16). A previous study discovered that phophorylation of $\mathrm{Bcl}-2$ at the evolutionarily conserved 

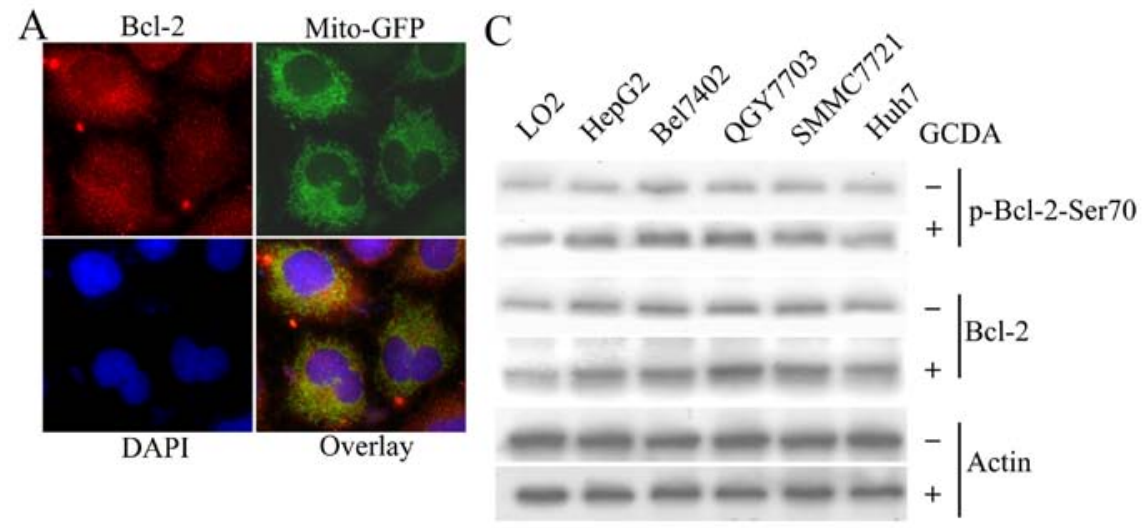

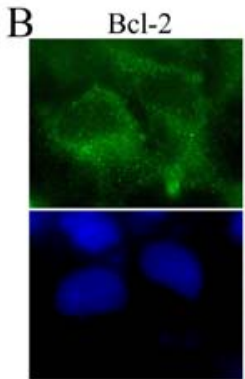

DAPI
ERM-RFP

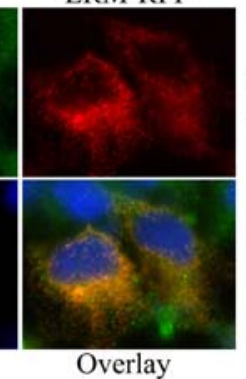

D

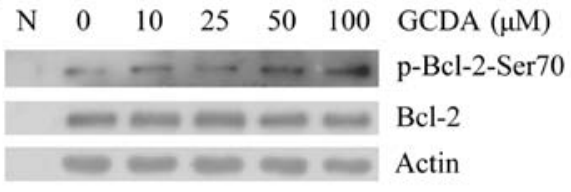

E

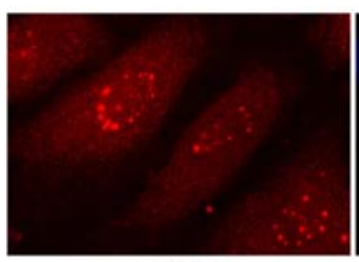

p-Bcl-2

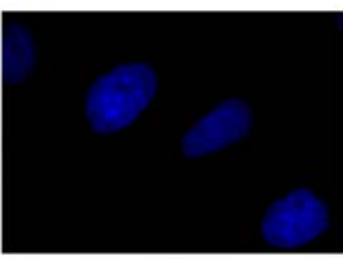

DAPI

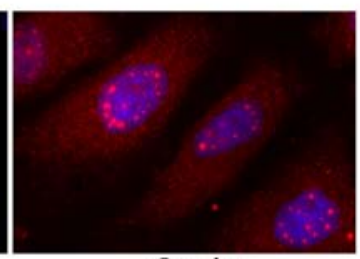

Overlay

Figure 3. GCDA stimulates Bcl-2 phosphorylation at Ser70. (A) Bcl-2 protein co-localizes with mitochondrial marker (Mito-GFP) in QGY7703 cells. (B) Bcl-2 protein co-localizes with endoplasmic reticulum marker (ERM-RFP) in QGY7703 cells. (C) HepG2, Bel7402, QGY7703, SMMC7721 and Huh7 cells were treated with $100 \mu \mathrm{M}$ GCDA for $30 \mathrm{~min}$. The expression of p-Bcl-2-Ser70 and Bcl2 was analyzed by western blot analysis. (D) QGY7703 cells were treated with gradually increasing doses of GCDA for $30 \mathrm{~min}$. The expression of p-Bcl-2-Ser70 and Bcl2 was analyzed by western blot analysis. (E) The subcellular localization of p-Bcl-2-Ser70 protein.

Ser70 site may be required for its full and potent antiapoptotic function (20,21). Therefore, we supposed that GCDA may induce the phosphorylation of Bcl-2 at Ser70 and then play an antiapoptotic effect. To test this, we treated HepG2, Bel7402, QGY7703, SMMC7721 and Huh7 cells with $100 \mu \mathrm{M}$ GCDA for $30 \mathrm{~min}$. Western blot analysis showed that the phosphorylation levels of Bcl-2 at Ser70, but not the expression levels of Bcl-2, increased in all the five cell lines after treated with GCDA (Fig. 3C). Furthermore, we treated QGY7703 cells with gradually increasing doses of GCDA for $30 \mathrm{~min}$. Western blot analysis showed that the phosphorylation levels of Bcl-2 at Ser70, but not the expression levels of Bcl-2, increased with the increasing GCDA concentrations (Fig. 3D). Notably, when Bcl-2 was phosphorylated at Ser70, abundant p-Bcl-2-Ser70 protein clustered in the nucleus, appearing as bigger spots in vision (Fig. 3E). These findings indicated that GCDA can induce the phosphorylation of Bcl-2 at Ser70 site. The p-Bcl2-Ser70 protein may function at the nucleus as polymers.

GCDA induces phosphorylation of Bcl-2 at Ser70 through $M A P K / E R K 1 / 2$ pathway. Previous research identified
ERK1/2 kinases as upstream Bcl-2 kinases (20). ERK1 and ERK2 can colocalize with mitochondrial Bcl-2 and directly phophorylate Bcl-2 on Ser-70 both in vitro and in vivo (20). To test whether GCDA-induced Bcl-2 phosphorylation occurs through MAPK/ERK1/2, QGY7703 cells were treated with increasing concentrations of GCDA for $30 \mathrm{~min}$. Phosphorylation of ERK1/2 was analyzed by western blot analysis using a phospho-specific ERK antibody. Results show that GCDA induces phosphorylation and activation of ERK1/2 in a dose-dependent manner (Fig. 4A). To further test whether inhibition of MAPK/ERK1/2 affects GCDA-induced Bcl-2 phosphorylation, QGY7703 cells expressing high levels of endogenous Bcl-2 were treated with GCDA in the absence or presence of increasing concentrations of PD98059 (MAPK/ERK1/2 inhibitor). Results suggest that PD98059 suppresses GCDA-stimulated phosphorylation of Bcl-2 at Ser70 and activation of ERK1/2, and has no effect on expression levels of endogenous Bcl-2 and ERK1/2 (Fig. 4B and C). QGY7703 cells were transfected with ERK1/2-siRNA or control-siRNA, phosphorylation of Bcl-2 at Ser70 and activation of ERK1/2 was analyzed by western 
A
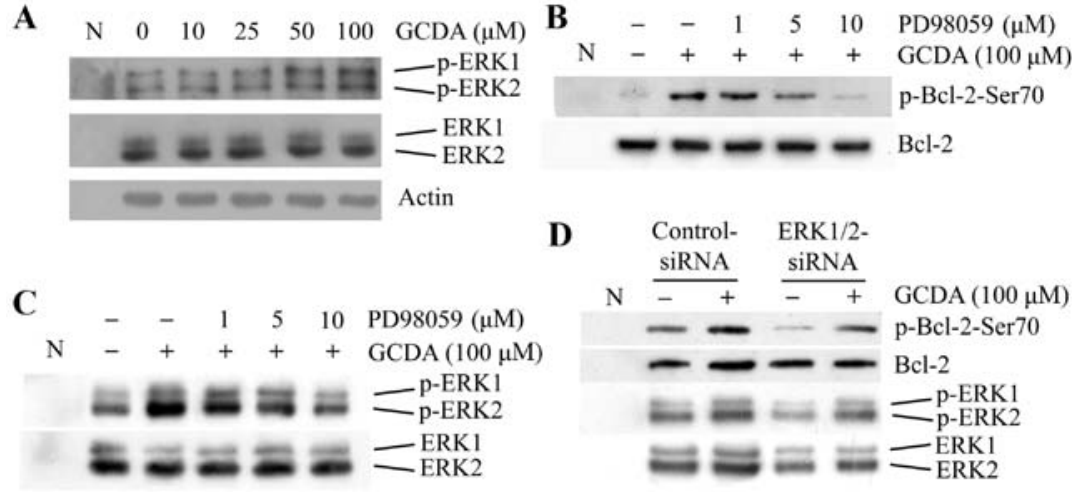

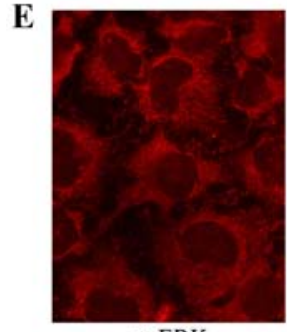

p-ERK

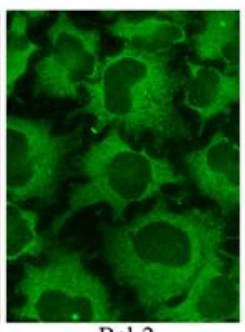

$\mathrm{Bcl}-2$

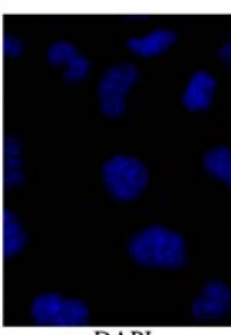

DAPI

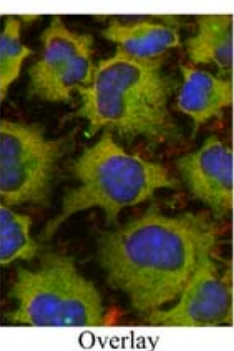

Overlay

Figure 4. GCDA induces phosphorylation of ERK1/2, which colocalizes with Bcl-2 in cytoplasm, and inhibition of ERK1/2 blocks GCDA-induced Bcl-2 phosphorylation at Ser70. (A) QGY7703 cells were treated with increasing concentrations of GCDA for 30 min. Western blot analysis was performed to detect the p-ERK1/2 using a phospho-specific ERK antibody. (B) QGY7703 cell were treated with GCDA (100 $\mu$ M) in the absence or presence of increasing concentrations of PD98059 for 30 min. Phosphorylation of Bcl-2 at Ser70 was determined by western blot analysis using a phospho-specific Bcl-2 antibody. (C) QGY7703 cell were treated with GCDA $(100 \mu \mathrm{M})$ in the absence or presence of increasing concentrations of PD98059 for 30 min. Phosphorylation of ERK1/2 was determined by western blot analysis using a phospho-specific ERK antibody. (D) ERK1/2 siRNA and control siRNA were transfected into QGY7703 cells using Lipofectamine 2000. After 24 h, cells were treated with or without GCDA (100 $\mu \mathrm{M})$ for 30 min. The level of p-Bcl-2-Ser70, Bcl-2, p-ERK1/2 and ERK1/2 were analyzed by western blot analysis using respective antibody. (E) p-ERK and Bcl-2 colocalized in QGY7703 cells. QGY7703 cell were fixed, incubated with a mouse p-ERK and a rabbit Bcl-2 primary antibody, and stained with Alexa Fluor ${ }^{\circledR} 594$ (red) donkey anti-mouse IgG (H+L) antibody and Alexa Fluor ${ }^{\circledR} 488$ (green) goat anti-rabbit IgG (H+L) antibody. Cells were mounted in MOWIOL R4-88 reagent (Calbiochem) and photographed with a Carl Zeiss AxioVision 4 microscope. N, negative control.
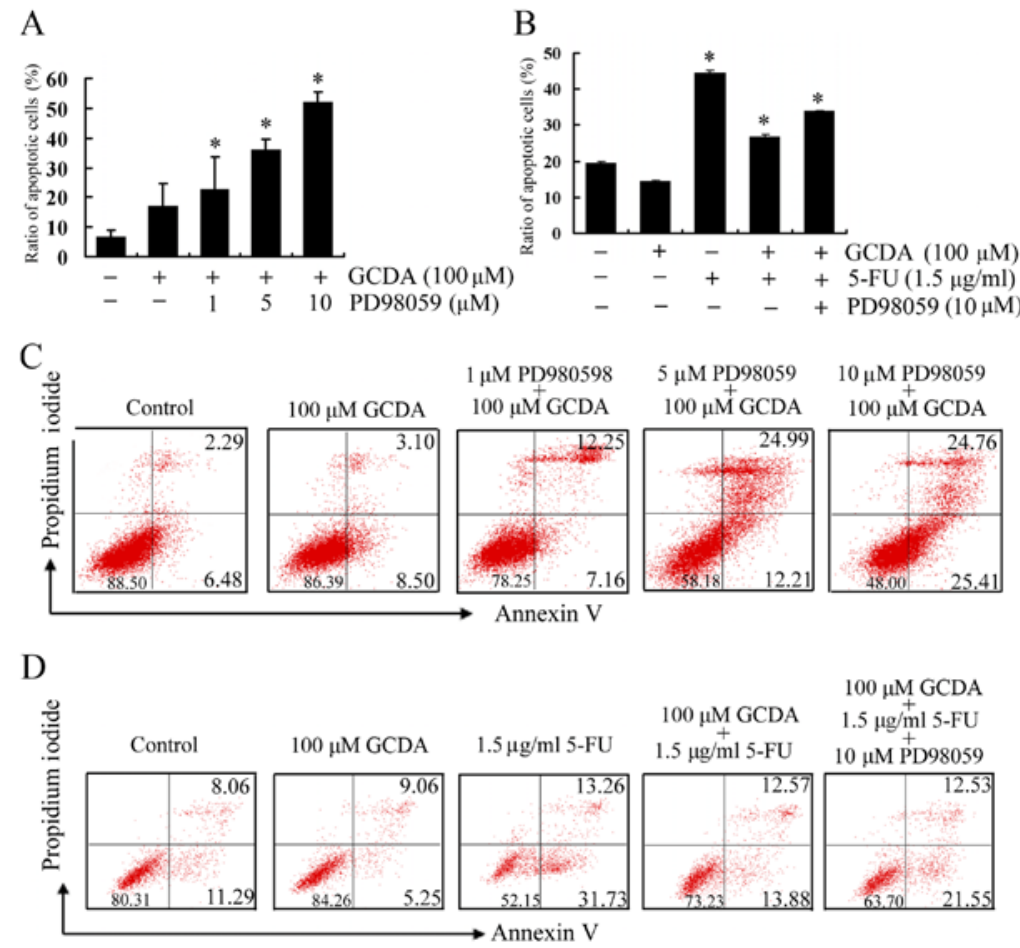

Figure 5. MAPK/ERK1/2 inhibitor PD98059 suppresses GCDA-induced survival and chemoresistance. (A and C) QGY7703 cells were treated with GCDA $(100 \mu \mathrm{M})$ in the absence or presence of increasing concentrations of PD98059 for 30 min. Cell apoptosis was determined by analyzing Annexin V binding on FASC. (B and D) QGY7703 cells were treated with 5-FU $(1.5 \mu \mathrm{g} / \mathrm{ml})$ and GCDA $(100 \mu \mathrm{M})$ in the absence or presence of increasing concentrations of PD98059 for $72 \mathrm{~h}$. Cell apoptosis was determined by analyzing Annexin V binding on FASC. Columns, means of three separate determinations; bars, SD; ${ }^{*}<0.05$ (Student's t-test). 


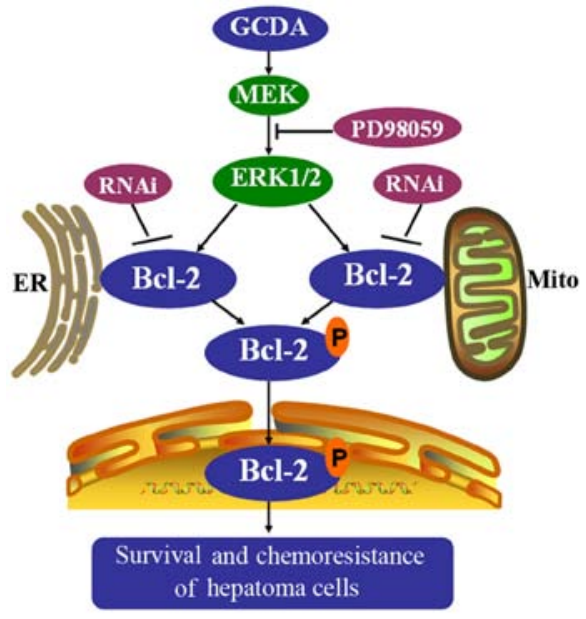

Figure 6. The signal pathway induced by GCDA mediates survival and chemoresistance of hepatocellular carcinoma. Glycochenodeoxycholate induces Bcl-2 phosphorylation at Ser70 site through activation of extracellular signalregulated kinase $1 / 2$ in association with increased chemoresistance of human liver cancer cells.

blot analysis using a phospho-specific Bcl-2 Ser70 and ERK antibody. Results indicate that knockdown of ERK1/2 protein expression by RNAi significantly decreases phosphorylation of Bcl-2 at Ser70 and activation of ERK1/2 (Fig. 4D). Coimmunofluorescent staining using p-ERK and Bcl-2 antibodies reveals that p-ERK and Bcl-2 colocalized in QGY7703 cells (Fig. 4E), which implies the direct interaction between p-ERK and Bcl-2. Thus, these findings suggest that GCDA-induced phosphorylation of Bcl-2 at Ser70 may occur through activation of ERK1/2.

The MAPK/ERK1/2 inhibitor PD98059 suppresses GCDAinduced survival and chemoresistance. The results above showed that PD98059 can decrease phosphorylation of Bcl-2 and ERK1/2, then whether PD98059 affects cell survival was assessed in QGY7703 cells expressing high levels of endogenous Bcl-2 treated with GCDA in the absence or presence of increasing concentrations of PD98059 for $30 \mathrm{~min}$. Cell viability and apoptosis were determined by analyzing Annexin V binding FACS. Results indicated that PD98059 mediated cell apoptosis in a dose-dependent manner (Fig. 5A and C). Next, to test whether PD98059 attenuates GCDA-induced chemoresistance, QGY7703 cells were treated with GCDA and 5-FU in the presence or absence of PD98059 for $72 \mathrm{~h}$. Annexin V binding FACS results showed that GCDA can prolong cell survival following treatment with 5-FU, and PD98059 significantly attenuated the chemoresistance of GCDA (Fig. 5B and D). Data represent the mean \pm SD of three determinations. $\mathrm{P}<0.05$ is based on the Student's t-test. These findings suggest that inhibition of MAPK/ERK1/2 pathway in human liver cancer cells may be pivotal for suppression of GCDA-induced chemoresistance.

\section{Discussion}

Bile acids are considered to be both detergent molecules that facilitate the solubilization of cheolesterol and absorption of lipids and regulatory molecules that activate five distinct receptors (farnesoid $\mathrm{X}$ receptor, pregnane $\mathrm{X}$ receptor, constitutive androstane receptor, vitamin $\mathrm{D}$ receptor and $\mathrm{G}$ protein-coupled receptor) and cell signaling pathways (c-jun $\mathrm{N}$-terminal kinase 1/2, AKT and ERK1/2) in cells in the liver and gastrointestinal tract $(38,39)$. Hepatocytes synthesize bile acids that secrete into the bile and are stored in the gallbladder (40). Eating food can stimulate bile acids to release into the intestinal tract where bile acids act as detergents to facilitate the solubilization of fatty acids and absorption of dietary lipids (40), then, bile acids are efficiently reabsorbed in the ileum and transported back to the liver via portal blood for resecretion into the bile. This process is known as enterohepatic circulation of bile acids (40). Recycling of bile acids/salts between the liver and intestine occurs 6-10 times each day and transports 20-40 g bile acids (39). Bile salts and bile acids are considered to be potential carcinogens. For example, bile acids play a role in colorectal carcinogenesis through ERKs and PKC signaling pathway (41); intrahepatic bile acid accumulation may have cocarcinogenic effects on the development of cholangiocarcinoma (42). Glycochenodeoxycholate (Glycine conjugate of chenodexycholate) is the main ingredient in the bile. Glycochenodeoxycholic acid can stimulate cell growth in Barrett's adenocarcinoma cell line (SEG-1) by activating the PI3 kinase/Akt signaling pathway (13), and induce the proliferation of non-neoplastic Barrett's cell line by activation of both ERK and p38 MAPK pathways (14). Hepatocellular carcinoma (HCC) is a highly malignant tumor that can evolve rapidly to acquire resistance to conventional chemotherapies (43). Our findings suggest that in all the five HCC cell lines, 5-FU can induce $~ 50 \%$ of cells to undergo apoptosis and GCDA can significantly attenuate the apoptotic effect of 5-FU (Fig. 1). These findings reveal that GCDA may be one reason of chemoresistance in liver cancer cells.

$\mathrm{Bcl}-2$ is the most important anti-apoptotic protein. Specific depletion of Bcl-2 from HepG2, QGY7703 and SMMC7721 cells by RNAi enhances sensitivity of those liver cancer cells to chemotherapeutic drug 5-FU, the chemoresistance effect of GCDA is downregulated (Fig. 2B-D). This suggests that $\mathrm{Bcl}-2$, which is an essential target for bile salt-induced chemoresistance in human liver cancer cells, should be a target for liver cancer treatment. At present, many inhibitors and microRNAs targeting Bcl-2 have been studied. miR-16 can inhibit glioma cell growth and invasion through the suppression of Bcl-2 (27). miR-18, a tumor-suppressive miRNA directly targeting Bcl-2, participated in suppression of cell proliferation and survival in nasopharyngeal carcinoma (29). MicroRNA-30b functions as a tumor suppressor in human colorectal cancer by targeting Bcl-2 (32). miR-449a could modulate cell cycle and apoptosis through regulating Bcl-2 expression in gastric cancer cell line SGC7901 (33). miR-210 can mediate hypoxia-induced neural apoptosis by targeting Bcl-2 (44). AT-101, a pan-Bcl-2 inhibitor, has high binding specificity for $\mathrm{Bcl}-2$ and $\mathrm{Mcl}-1$ and potentiates the cytotoxic effects of lenalidomide and dexamethasone in preclinical models of plasma cell cancers (multiple myeloma and Waldenstrom macroglobulinaemia) (28). ABT-737, which is an anticancer drug, is a Bcl-2 Homology 3 (BH-3)-mimetic that induces apoptosis by inhibiting pro-survival $\mathrm{Bcl}-2$ proteins (45) and enhance the effects of epirubicin on HepG2 cells by activating autophagy and inducing apoptosis (46). 
A BH3 mimetic, ABT-199, has been developed to selectively bind Bcl-2 and enhances imatinib-induced cell death in chronic myeloid leukemia progenitors (47). BDA-366, a small-molecule Bcl-2-BH4 domain antagonist, can bind BH4 of Bcl-2 with high affinity and selectivity, and this Bcl-2-BH4 antagonist may provide a strategy to improve lung cancer outcome (48).

Bcl-2 is an anti-apototic molecule, whose phosphoylation at Ser70 by growth factor-activated protein kinases including PKC and the MAPKs (ERK1/2) can positively regulate the anti-apoptotic function of Bcl-2 (20,49,50). Our findings indicate that $\mathrm{Bcl}-2$ is expressed in all the five liver cancer cell lines (Fig. 2A). Glycochenodeoxycholate (GCDA), the main ingredient in the bile, can mimic growth factor to stimulate Bcl-2 phosphorylation at Ser70 site in dose-dependent manner (Fig. 3D), which is associated with enhanced chemoresistance of liver cancer cells (Figs. 1 and 5B). ERK1 and ERK2 are physiologic Bcl-2 kinases that can phosphorylate Bcl-2 at Ser70 site. Intriguingly, GCDA can stimulate phosphorylation and activation of ERK1/2 (Fig. 4A). Activated ERK1/2 (p-ERK1/2) can colocalize with Bcl-2 in cytoplasm (Fig. 4E). Because both the specific MEK/ERK inhibitor PD 98059 and siRNA targeting ERK1/2 block GCDAstimulated phosphorylation of ERK1/2 and Bcl-2 (Fig. 4B-D) and attenuates GCDA-induced survival and chemoresistance (Fig. 5A and B), we propose that ERK1 and ERK2 function as GCDA-activated Bcl-2 kinases to phosphorylate Bcl-2 and regulate its activity in human liver cancer cells.

Bcl-2 at the ER is required in cancer cells, such cells will be sensitive to BH4-domain targeting drugs (16). Our results showed that in QGY7703 cells Bcl-2 localized both at mitochondria and endoplasmic reticulum (Fig. 3A and B), which indicated that $\mathrm{Bcl}-2$ may prevent apoptosis by disturbing Bim-mediated Bax/Bak activation or toxic $\mathrm{Ca}^{2+}$ release. In addition, the function of $\mathrm{Bcl}-2$ at the nucleus is not so clear. Phosphorylation of Bcl-2 at Ser70 site induced by NNK may facilitate the direct interaction between $\mathrm{Bcl}-2$ and c-Myc in the nucleus that enhances the half-life of c-Myc and finally promotes cell survival and chemoresistance in human lung cancer (51). NNK can induce the accumulation of Bcl-2 in the nucleus, which disrupts the hMSH2-hMSH6 complex and suppresses DNA mismatch repair in vitro (52). Besides, the accumulation of Bcl-2 in the nucleus induced by NNK can also interact with APE1, which disrupts APE1·XRCC1 complex with suppresion of APE1 endonulease activity and AP site repair (53). Our results show that when Bcl-2 was phosphorylated at Ser70, abundant p-Bcl-2-Ser70 protein cluster in the nucleus, appearing as bigger spots (Fig. 3E). The p-Bcl-2-Ser70 protein may function at the nucleus as polymers. However, the $\mathrm{p}-\mathrm{Bcl}-2-\mathrm{Ser} 70$ protein participation in the nucleus in transcriptional regulation or DNA repair need to be further studied.

In summary, the present study found that glycochenodeoxycholate (GCDA) induces chemoresistance of human liver cancer cells by activating the anti-apoptotic function of Bcl-2 via its phosphorylation. GCDA stimulates Bcl-2 phosphorylation at Ser70 site in the flexible loop domain through activating ERK1/2. GCDA-induced Bcl-2 phosphorylation at Ser70 site leads to its chemoresistance of human liver cancer cells (Fig. 6). Thus, disruption of the anti-apoptotic function of
Bcl-2 by blocking its Ser70 site phosphorylation may represent a strategy for the treatment of GCDA-induce chemoresistance in human liver cancers.

\section{Acknowledgements}

The present study was supported by the National Natural Science Foundation of China (nos. 81402001, 81272193 and 81302075), the Natural Science Foundation of Hunan Province (no. 2016JJ3177) and the Young Teachers Boost Project of Central South University (2012QNZT114). We thank Dr Yuan (Sun Yat-sen University) for kindly providing mitochondrial marker (Mito-GFP) and endoplasmic reticulum marker (ERM-RFP) plasmids.

\section{References}

1. Chen W, Zheng R, Baade PD, Zhang S, Zeng H, Bray F, Jemal A, Yu XQ and He J: Cancer statistics in China, 2015. CA Cancer J Clin 66: 115-132, 2016.

2. Allemani C, Weir HK, Carreira H, Harewood R, Spika D, Wang XS, Bannon F, Ahn JV, Johnson CJ, Bonaventure A, et al; CONCORD Working Group: Global surveillance of cancer survival 1995-2009: Analysis of individual data for 25,676,887 patients from 279 population-based registries in 67 countries (CONCORD-2). Lancet 385: 977-1010, 2015.

3. Faubion WA, Guicciardi ME, Miyoshi H, Bronk SF, Roberts PJ, Svingen PA, Kaufmann SH and Gores GJ: Toxic bile salts induce rodent hepatocyte apoptosis via direct activation of Fas. J Clin Invest 103: 137-145, 1999.

4. Sodeman T, Bronk SF, Roberts PJ, Miyoshi H and Gores GJ: Bile salts mediate hepatocyte apoptosis by increasing cell surface trafficking of Fas. Am J Physiol Gastrointest Liver Physiol 278: G992-G999, 2000.

5. Jones BA, Rao YP, Stravitz RT and Gores GJ: Bile salt-induced apoptosis of hepatocytes involves activation of protein kinase $\mathrm{C}$. Am J Physiol 272: G1109-G1115, 1997.

6. Gonzalez B, Fisher C and Rosser BG: Glycochenodeoxycholic acid (GCDC) induced hepatocyte apoptosis is associated with early modulation of intracellular PKC activity. Mol Cell Biochem 207: 19-27, 2000.

7. Tsuchiya S, Tsuji M, Morio Y and Oguchi K: Involvement of endoplasmic reticulum in glycochenodeoxycholic acid-induced apoptosis in rat hepatocytes. Toxicol Lett 166: 140-149, 2006

8. Drudi Metalli V, Mancino MG, Mancino A, Torrice A, Gatto M, Attili AF, Alpini G and Alvaro D: Bile salts regulate proliferation and apoptosis of liver cells by modulating the IGF1 system. Dig Liver Dis 39: 654-662, 2007.

9. Wang K, Brems JJ, Gamelli RL and Ding J: Reversibility of caspase activation and its role during glycochenodeoxycholateinduced hepatocyte apoptosis. J Biol Chem 280: 23490-23495, 2005.

10. Wang K, Brems JJ, Gamelli RL and Holterman AX: Survivin signaling is regulated through nuclear factor-kappa B pathway during glycochenodeoxycholate-induced hepatocyte apoptosis. Biochim Biophys Acta 1803: 1368-1375, 2010.

11. Webster CR, Johnston AN and Anwer MS: Protein kinase CD protects against bile acid apoptosis by suppressing proapoptotic JNK and BIM pathways in human and rat hepatocytes. Am J Physiol Gastrointest Liver Physiol 307: G1207-G1215, 2014.

12. Yoon JH, Werneburg NW, Higuchi H, Canbay AE, Kaufmann SH, Akgul C, Edwards SW and Gores GJ: Bile acids inhibit Mcl-1 protein turnover via an epidermal growth factor receptor/Raf-1dependent mechanism. Cancer Res 62: 6500-6505, 2002.

13. Jaiswal K, Tello V, Lopez-Guzman C, Nwariaku F, Anthony T and Sarosi GA Jr: Bile salt exposure causes phosphatidyl-inositol3-kinase-mediated proliferation in a Barrett's adenocarcinoma cell line. Surgery 136: 160-168, 2004.

14. Jaiswal K, Lopez-Guzman C, Souza RF, Spechler SJ and Sarosi GA Jr: Bile salt exposure increases proliferation through p38 and ERK MAPK pathways in a non-neoplastic Barrett's cell line. Am J Physiol Gastrointest Liver Physiol 290: G335-G342, 2006. 
15. Komichi D, Tazuma S, Nishioka T, Hyogo H and Chayama K: Glycochenodeoxycholate plays a carcinogenic role in immortalized mouse cholangiocytes via oxidative DNA damage. Free Radic Biol Med 39: 1418-1427, 2005.

16. Akl H, Vervloessem T, Kiviluoto S, Bittremieux M, Parys JB, De Smedt $\mathrm{H}$ and Bultynck G: A dual role for the anti-apoptotic $\mathrm{Bcl}-2$ protein in cancer: Mitochondria versus endoplasmic reticulum. Biochim Biophys Acta 1843: 2240-2252, 2014.

17. Tsujimoto Y: Bcl-2 family of proteins: Life-or-death switch in mitochondria. Biosci Rep 22: 47-58, 2002.

18. Cory S and Adams JM: The Bcl2 family: Regulators of the cellular life-or-death switch. Nat Rev Cancer 2: 647-656, 2002.

19. Deng X, Gao F, Flagg T and May WS Jr: Mono- and multisite phosphorylation enhances Bcl2's antiapoptotic function and inhibition of cell cycle entry functions. Proc Natl Acad Sci USA 101: 153-158, 2004.

20. Deng X, Ruvolo P, Carr B and May WS Jr: Survival function of ERK1/2 as IL-3-activated, staurosporine-resistant Bcl2 kinases. Proc Natl Acad Sci USA 97: 1578-1583, 2000.

21. Deng X, Xiao L, Lang W, Gao F, Ruvolo P and May WS Jr: Novel role for JNK as a stress-activated Bcl2 kinase. J Biol Chem 276 23681-23688, 2001

22. Zhang C, Wu Z, Hong W, Wang Z, Peng D, Chen J, Yuan C, Yu S, $\mathrm{Xu}$ L and Fang Y: Influence of BCL2 gene in major depression susceptibility and antidepressant treatment outcome. J Affect Disord 155: 288-294, 2014.

23. Moreno-Galindo C, Hermsen M, García-Pedrero JM, Fresno MF, Suárez C and Rodrigo JP: p27 and BCL2 expression predicts response to chemotherapy in head and neck squamous cell carcinomas. Oral Oncol 50: 128-134, 2014.

24. Xu P, Liu L, Wang J, Zhang K, Hong X, Deng Q, Xiang J, Zhang X, He M, Wu T, et al: Genetic variation in BCL2 3'-UTR was associated with lung cancer risk and prognosis in male Chinese population. PLoS One 8: e72197, 2013.

25. Mehta SV, Shukla SN and Vora HH: Overexpression of $\mathrm{Bcl} 2$ protein predicts chemoresistance in acute myeloid leukemia: Its correlation with FLT3. Neoplasma 60: 666-675, 2013.

26. Dorjgochoo T, Xiang YB, Long J, Shi J, Deming S, Xu WH Cai H, Cheng J, Cai Q, Zheng W, et al: Association of genetic markers in the BCL-2 family of apoptosis-related genes with endometrial cancer risk in a Chinese population. PLoS One 8: e60915, 2013.

27. Yang TQ, Luo XJ, Wu TF, Ding DD, Zhao ZH, Chen GL, Xie XS, Li B, Wei YX, Guo LC, et al: MicroRNA-16 inhibits glioma cell growth and invasion through suppression of BCL2 and the nuclear factor- $\kappa$ B1/MMP9 signaling pathway. Cancer Sci 105 : 265-271, 2014.

28. Paulus A, Chitta K, Akhtar S, Personett D, Miller KC, Thompson KJ, Carr J, Kumar S, Roy V, Ansell SM, et al: AT-101 downregulates BCL2 and MCL1 and potentiates the cytotoxic effects of lenalidomide and dexamethasone in preclinical models of multiple myeloma and Waldenström macroglobulinaemia. $\mathrm{Br}$ J Haematol 164: 352-365, 2014

29. Zhen Y, Liu Z, Yang H, Yu X, Wu Q, Hua S, Long X, Jiang Q, Song Y, Cheng C, et al: Tumor suppressor PDCD4 modulates miR-184-mediated direct suppression of C-MYC and BCL2 blocking cell growth and survival in nasopharyngeal carcinoma. Cell Death Dis 4: e872, 2013

30. Yao F, Han Q, Zhong $\mathrm{C}$ and Zhao H: TRAF6 promoted the tumorigenicity of esophageal squamous cell carcinoma. Tumour Biol 34: 3201-3207, 2013 .

31. Petrasek J, Iracheta-Vellve A, Csak T, Satishchandran A, Kodys K, Kurt-Jones EA, Fitzgerald KA and Szabo G: STING-IRF3 pathway links endoplasmic reticulum stress with hepatocyte apoptosis in early alcoholic liver disease. Proc Natl Acad Sci USA 110: 16544-16549, 2013

32. Liao WT, Ye YP, Zhang NJ, Li TT, Wang SY, Cui YM, Qi L, Wu P, Jiao HL, Xie YJ, et al: MicroRNA-30b functions as a tumour suppressor in human colorectal cancer by targeting KRAS, PIK3CD and BCL2. J Pathol 232: 415-427, 2013.

33. Hu J, Fang Y, Cao Y, Qin R and Chen Q: miR-449a regulates proliferation and chemosensitivity to cisplatin by targeting cyclin D1 and BCL2 in SGC7901 cells. Dig Dis Sci 59: 336-345, 2013.
34. Drukker L, Margulis A, Chaouat M, Levitzki R, Maiorenko E and Ben Bassat H: Changes of PI3K/AKT/BCL2 signaling proteins in congenital Giant Nevi: Melanocytes contribute to their increased survival and integrity. J Recept Signal Transduct Res 33: 359-366, 2013

35. Kurosawa H, Que FG, Roberts LR, Fesmier PJ and Gores GJ: Hepatocytes in the bile duct-ligated rat express Bcl-2. Am J Physiol 272: G1587-G1593, 1997.

36. Wang $J$ and Zou S: The bcl-2 mRNA expression in GCDCinduced obstructive jaundice in rats and its implication in hepatocellular apoptosis. J Huazhong Univ Sci Technolog Med Sci 22: 34-36, 2002.

37. Liao M, Zhao J, Wang T, Duan J, Zhang Y and Deng X: Role of bile salt in regulating Mcl-1 phosphorylation and chemoresistance in hepatocellular carcinoma cells. Mol Cancer 10: 44, 2011.

38. Hylemon PB, Zhou H, Pandak WM, Ren S, Gil G and Dent P: Bile acids as regulatory molecules. J Lipid Res 50: 1509-1520, 2009.

39. Sipka S and Bruckner G: The immunomodulatory role of bile acids. Int Arch Allergy Immunol 165: 1-8, 2014.

40. Li T and Chiang JY: Bile acid signaling in metabolic disease and drug therapy. Pharmacol Rev 66: 948-983, 2014.

41. Debruyne PR, Bruyneel EA, Li X, Zimber A, Gespach C and Mareel MM: The role of bile acids in carcinogenesis. Mutat Res 480-481: 359-369, 2001.

42. Lozano E, Sanchez-Vicente L, Monte MJ, Herraez E, Briz O, Banales JM, Marin JJ and Macias RI: Cocarcinogenic effects of intrahepatic bile acid accumulation in cholangiocarcinoma development. Mol Cancer Res 12: 91-100, 2014.

43. Yang X, Sun D, Tian Y, Ling S and Wang L: Metformin sensitizes hepatocellular carcinoma to arsenic trioxide-induced apoptosis by downregulating Bcl2 expression. Tumour Biol 36: 2957-2964, 2015.

44. Chio CC, Lin JW, Cheng HA, Chiu WT, Wang YH, Wang JJ, Hsing $\mathrm{CH}$ and Chen RM: MicroRNA-210 targets antiapoptotic $\mathrm{Bcl}-2$ expression and mediates hypoxia-induced apoptosis of neuroblastoma cells. Arch Toxicol 87: 459-468, 2013.

45. Rooswinkel RW, van de Kooij B, Verheij M and Borst J: Bcl-2 is a better ABT-737 target than Bcl-xL or Bcl-w and only Noxa overcomes resistance mediated by Mcl-1, Bfl-1, or Bcl-B. Cell Death Dis 3: e366, 2012.

46. Du P, Cao H, Wu HR, Zhu BS, Wang HW, Gu CW, Xing CG and Chen W: Blocking Bcl-2 leads to autophagy activation and cell death of the HEPG2 liver cancer cell line. Asian Pac J Cancer Prev 14: 5849-5854, 2013.

47. Ko TK, Chuah CT, Huang JW, Ng KP and Ong ST: The BCL2 inhibitor ABT-199 significantly enhances imatinib-induced cell death in chronic myeloid leukemia progenitors. Oncotarget 5: 9033-9038, 2014

48. Han B, Park D, Li R, Xie M, Owonikoko TK, Zhang G, Sica GL, Ding C, Zhou J, Magis AT, et al: Small-molecule Bcl2 BH4 antagonist for lung cancer therapy. Cancer Cell 27: 852-863, 2015.

49. Mai H, May WS, Gao F, Jin Z and Deng X: A functional role for nicotine in $\mathrm{Bcl} 2$ phosphorylation and suppression of apoptosis. J Biol Chem 278: 1886-1891, 2003.

50. Ruvolo PP, Deng X, Carr BK and May WS: A functional role for mitochondrial protein kinase Calpha in $\mathrm{Bcl} 2$ phosphorylation and suppression of apoptosis. J Biol Chem 273: 25436-25442, 1998.

51. Jin Z, Gao F, Flagg T and Deng X: Tobacco-specific nitrosamine 4-(methylnitrosamino)-1-(3-pyridyl)-1-butanone promotes functional cooperation of $\mathrm{Bcl} 2$ and $\mathrm{c}-\mathrm{Myc}$ through phosphorylation in regulating cell survival and proliferation. J Biol Chem 279: 40209-40219, 2004.

52. Hou Y, Gao F, Wang Q, Zhao J, Flagg T, Zhang Y and Deng X: Bcl2 impedes DNA mismatch repair by directly regulating the hMSH2-hMSH6 heterodimeric complex. J Biol Chem 282: 9279-9287, 2007.

53. Zhao J, Gao F, Zhang Y, Wei K, Liu Y and Deng X: Bcl2 inhibits abasic site repair by down-regulating APE1 endonuclease activity. J Biol Chem 283: 9925-9932, 2008. 